\title{
THE NUCLEOLUS OF A MATRIX GAME AND OTHER NUCLEOLI*
}

\author{
JOS A. M. POTTERS AND STEF H. TIJS
}

\begin{abstract}
We define the nucleolus of a continuous convex map $F: \Pi \rightarrow \mathbf{R}^{m}$ on a convex compact set $\Pi \subset \mathbf{R}^{n}$. As special cases we obtain known notions as nucleolus, prenucleolus and weighted nucleolus of a TU-game with (or without) coalition structure. Also the nucleolus of a matrix game turns out to be an interesting special case. It appears that the nucleolus of a matrix game coincides with the set of Dresher optimal strategy pairs of the game. This implies, in particular, that the nucleolus consists of the proper equilibria of the matrix game. To each (zero-normalized) TU-game one can construct a matrix game-the excess game-such that the nucleolus of the TU-game coincides with the unique proper optimal strategy of player II in the excess game. Also for other nucleoli of TU-games a suitable matrix game can be constructed where the nucleolus under consideration is related to the nucleolus of the matrix game in an analogous way. A balancedness condition is given characterizing nucleolus elements of a matrix game. It is shown that this balancedness result implies the known balancedness characterizations of Kohlberg, Sobolev, Owen and Wallmeier.
\end{abstract}

0. Introduction. In Dresher (1961) an interesting multi-step procedure is described to select from the optimal strategy set of a player in a matrix game a subset of optimal strategies which exploit mistakes of the opponent optimally. This Dresher procedure which refines the set of equilibrium points of a matrix game turns out to be equal to the refinement of Myerson (1978) as it is shown in van Damme (1983). So the set of Dresher saddle points of a matrix game form the set of proper equilibria of the game. Later on we will call this set-and for good reasons - the nucleolus of the matrix game. The elements in the nucleolus of a player in a matrix game can be characterized by an interesting balancedness property. This characterization is obtained by using an old result of Bohnenblust et al. (1950) (cf. Gale and Sherman 1950) which states that the set of equalizers of a player coincides with the set of all pure strategies used in at least one optimal strategy.

The nucleoli of coalitional games (Schmeidler 1969, Grotte 1970, Sobolev 1975, Owen 1977) are closely related to the nucleolus of well-chosen matrix games. More precisely, one can construct to a 0 -normalized $n$-person coalitional game a $\left(2^{n}-2\right) \times n$ matrix game - the excess game-in such a way that, e.g., the nucleolus of Schmeidler (1969) is the unique Dresher optimal strategy of player II in the excess game. The strategy set of player II in the excess game is just the set of imputations of the coalitional game. The columns of the excess game have the excesses of the extreme points of the imputation set as coordinates. Also for the prenucleolus and weighted nucleolus of a coalitional game with or without coalition structure one can define suitable excess games. From this correspondence between the nucleoli of coalitional games and the nucleolus of suitably chosen matrix game it is easy to interpret the balancedness results for matrix games in the case of coalitional games

${ }^{*}$ Received May 12, 1989; revised July 16, 1990.

AMS 1980 subject classification. Primary: 90D12.

LAOR 1973 subject classification. Main: Games.

OR/MS Index 1978 subject classification. Primary: 234 Games/cooperative.

Key words. Matrix games, excess game, nucleolus, optimal strategy. 
and to prove the results of Kohlberg (1971), Sobolev (1975), Owen (1977) and Wallmeier (1980).

The paper is organized as follows. First we define the most abstract concept-the nucleolus of a convex continuous map of a compact convex subset of $\mathbf{R}^{n}$ into $\mathbf{R}^{m}$-and describe a procedure for finding the nucleolus. Using this concept it is easy to introduce in a unified way the nucleolus of a matrix game and the nucleoli of coalitional game. The "nucleolus hunting" procedure for convex maps coincides with the Dresher procedure in case of a matrix game. Next we derive the balancedness condition for matrix games and the relation with the set of proper equilibria. Finally, we construct to the different nucleoli of coalitional games their excess games and use the results of the nucleolus of a matrix game to obtain results in cooperative game theory.

1. The nucleolus $N(\Pi, F)$. In this section we define the central concept of this paper-the nucleolus.

Let $\Pi$ be a nonempty compact convex subset of $\mathbf{R}^{n}$ and $F: \Pi \rightarrow \mathbf{R}^{m}$ a continuous convex map. The following two properties are easy to prove.

- If $\mathscr{B} \subset \mathbf{N}_{m}$ then the function $g(x)=\max \left\{F_{j}(x) \mid j \in \mathscr{B}\right\}$ is continuous and convex.

- If $\mathscr{B} \subset \mathbf{N}_{m}$ and $w \in \mathbf{R}$ such that $F_{j}(x) \leqslant w$ for all $x \in \Pi$ and for each (1.2) $j \in \mathscr{B}$ there is a point $x_{j} \in \Pi$ where $F_{j}\left(x_{j}\right)<w$, then $F_{j}(x)<w$ for all $j \in \mathscr{B}$ for some point $x \in \Pi$ (e.g., the barycenter of the points $\left\{x_{j}\right\}_{j \in \mathscr{B}}$ ).

For any $m \in \mathbf{N}$ we define the map $\theta: \mathbf{R}^{m} \rightarrow \mathbf{R}^{m}$ which orders the coordinates of a point of $\mathbf{R}^{m}$ in a weakly decreasing order, i.e., $\theta(x)=y$ iff there is a permutation $\tau$ of $\mathbf{N}_{m}=\{1, \ldots, m\}$ such that $y_{i}=x_{\tau(i)}$ for all $i \in \mathbf{N}_{m}$ and $y_{1} \geqslant y_{2} \geqslant \cdots \geqslant y_{m}$.

Further, we define the lexicographic order $\preceq_{\text {lex }}$ by $x \preceq_{\text {lex }} y$ iff there is an index $k \leqslant m$ such that $x_{i}=y_{i}$ for all $i<k$ and $x_{k}<y_{k}$ or $x=y$.

The following properties are well known:

(1.3) - The lexicographic order $\preceq_{\text {lex }}$ is transitive, complete and asymmetric.

(1.4) - If $x, y \in \mathbf{R}^{m}, z \in \mathbf{R}^{p}$ then $\theta(x) \preceq_{\operatorname{lex}} \theta(y) \Leftrightarrow \theta(x, z) \preceq_{\operatorname{lex}} \theta(y, z)$.

We define the nucleolus $N(\Pi, F)$ by

$$
N(\Pi, F):=\left\{x \in \Pi \mid \theta \circ F(x) \preceq_{\operatorname{lex}} \theta \circ F(y) \text { for all } y \in \Pi\right\} .
$$

TheOrem 1. The nucleolus $N(\Pi, F)$ is a nonempty compact convex subset of $\Pi$ and the map $F$ is constant on $N(\Pi, F)$.

Proof. In this proof we make the following construction to be used in the sequel many times. Let $\Pi_{0}=\Pi$ and $\mathscr{B}_{0}=\varnothing$. If $\mathscr{B}_{i-1} \neq \mathbf{N}_{m}$ we define inductively $g_{i}:=$ $\max \left\{F_{j} \mid j \notin \mathscr{B}_{i-1}\right\}$, a continuous and convex function (by (1.1)), $w_{i}:=\min \left\{g_{i}(x) \mid x \in\right.$ $\left.\Pi_{i-1}\right\}$, well-defined by the continuity of $g_{i}, \Pi_{i}:=\left\{x \in \Pi_{i-1} \mid g_{i}(x)=w_{i}\right\}$, the set of points where $g_{i} \mid \Pi_{i-1}$ attains its minimal value; the set $\Pi_{i}$ is compact (by the continuity of $g_{i}$ ) and convex (by the convexity of $\left.g_{i}\right), \mathscr{B}_{i}:=\left\{j \in \mathbf{N}_{m} \mid F_{j}(x) \geqslant w_{i}\right.$ for all $\left.x \in \Pi_{i}\right\}$.

We can make the following observations:

- For all $j \notin \mathscr{B}_{i-1}$ there is a point $x_{j} \in \Pi_{i-1}$ such that $F_{j}\left(x_{j}\right)<w_{i-1}$ and $F_{j}\left(x_{k}\right) \leqslant$ $w_{i-1}$ for all $k \notin \mathscr{B}_{i-1}, k \neq j$. Then $F_{j}(x)<w_{i-1}$ for all $j \notin \mathscr{B}_{i-1}$ if $x$ is the 
barycenter of the point $x_{j}, j \notin \mathscr{B}_{i-1}$ (by (1.2)). This means that $w_{i}<w_{i-1}$ and $\mathscr{B}_{i} \supset \mathscr{B}_{i-1}$.

- Suppose that to every $j \notin \mathscr{B}_{i-1}$ there is a point $x_{j} \in \Pi_{i}$ such that $F_{j}\left(x_{j}\right)<w_{i}$. Then $F_{j}(x)<w_{i}$ for all $j \notin \mathscr{B}_{i-1}$ if $x$ is the barycenter of the points $x_{j}, j \notin \mathscr{B}_{i-1}$ (by (1.2)) and hence $g_{i}(x)<w_{i}$ and $x \in \Pi_{i}$ in contradiction with the definition of $w_{i}$. So, there is an index $j \in \mathscr{B}_{i} \backslash \mathscr{B}_{i-1}$.

- Hence, eventually we have $\mathscr{B}_{s}=\mathbf{N}_{m}$ for some $s \in \mathbf{N}$.

We shall prove that $\Pi_{s}=N(\Pi, F)$.

- We prove that for $i=1, \ldots, s$, if $x \in \Pi_{i}$ and $\theta \circ F(y) \preceq_{\operatorname{lex}} \theta \circ F(x)$, then $y \in \Pi_{i}$. First we notice that for all $j \in \mathscr{B}_{i} \backslash \mathscr{B}_{i-1}$ and $x \in \Pi_{i}$, we have $F_{j}(x) \geqslant w_{i}$ and by the definition of $\Pi_{i}$ we have $F_{j}(x)=w_{i}$. Suppose that $y \in \Pi_{k-1}$ with $k<i$. Then $F_{j}(x)=F_{j}(y)$ for all $j \in \mathscr{B}_{k-1}$ and $g_{k}(y) \leqslant g_{k}(x)=w_{k}$ (by (1.4)). Since $w_{k}=$ $\min \left\{g_{k}(x) \mid x \in \Pi_{k-1}\right\}$ we have equality and $y \in \Pi_{k}$. Since we can repeat this argument while $k \leqslant i$ we find finally $y \in \Pi_{i}$.

- For $i=s$ we find that, if $x \in \Pi_{s}$ and $\theta \circ F(y) \preceq_{\operatorname{lex}} \theta \circ F(x)$, then $y \in \Pi_{s}$ and $F_{j}(y)=F_{j}(x)=w_{k}(j)$ for all $j \in \mathbf{N}_{m}$ where $k(j)$ is determined by $j \in \mathscr{B}_{k(j)} \backslash \mathscr{B}_{k(j)-1}$. This means that $F(x)=F(y)$ and that there is no point $y \in \Pi$ such that $\theta$ 。 $F(y) \prec{ }_{\text {lex }} \theta \circ F(x): \Pi_{s} \subset N(\Pi, F)$.

- If conversely $y \in N(\Pi, F)$ and $x \in \Pi_{s}$ then $\theta \circ F(y) \preceq_{\operatorname{lex}} \theta \circ F(x)$ gives $y \in \Pi_{s}$.

Conclusion: $\Pi_{s}=N(\Pi, F)$ is nonempty convex and compact and if $x \in N(\Pi, F)$ then $N(\Pi, F)=\{y \in \Pi \mid F(y)=F(x)\}$. QED

For use later on we recall that, for $i=1, \ldots, s$,

$$
\Pi_{i}=\left\{x \in \Pi \mid F_{j}(x)=w_{k(j)} \text { for all } j \in \mathscr{B}_{i}\right\}
$$

where $k(j)=\min \left\{k \in \mathbf{N} \mid j \in \mathscr{B}_{k}\right\}$ is.

For an axiomatic characterization of the nucleolus correspondence we refer to Maschler, Potters and Tijs (1991).

The following examples play a crucial role in the remaining sections.

EXAMPLE 1. The nucleolus of a two-person zero-sum game. Let $A$ be an $m \times n$ matrix and $M(N)$ the set of rows (columns) of $A$. Let $\Delta_{I}$ and $\Delta_{I I}$ be set of probability measures on $M$ and $N$.

If we take $\Pi=\Delta_{I}$ and $F=\left\{F_{j}: p \rightarrow-p A e_{j}, j \in N\right\}$ then $N_{I}(A):=N(\Pi, F)$.

If we take $\Pi=\Delta_{I I}$ and $F=\left\{F_{i}: q \rightarrow e_{i} A q, i \in M\right\}$ then $N_{I I}(A):=N(\Pi, F)$.

Finally we define $N(A):=N_{I}(A) \times N_{I I}(A)$-the nucleolus of the matrix game $A$.

In the next section we shall prove that $N(A)$ is the set of proper equilibria of the matrix game $A$.

EXAMPLE 2. The nucleoli of a cooperative game with side payments. (a) Let $(N, v)$ be a game in characteristic function form. If we take $\Pi=I(v)$, the set of imputations of the game $(N, v)$ and $F=\left\{F_{S}\right\}_{S \subset N, S \neq N, \varnothing}$ where $F_{S}$ is the excess function of coalition $S$ defined by $F_{S}(x):=v(S)-\sum_{i \in S} x_{i}$ for all points $x \in I(v)$, then we find the nucleolus of the game $N(v)$ as introduced in Schmeidler (1969).

(b) Let $(N, v)$ be a cooperative game and $\mathscr{T}=\left\{T_{1}, \ldots, T_{k}\right\}$ be a partition of $N$ (i.e., a coalition structure). We take

$$
\begin{aligned}
\Pi & =I(v, \mathscr{T}) \\
& :=\left\{x \in \mathbf{R}^{n} \mid x\left(T_{i}\right)=v\left(T_{i}\right) \text { for } i=1, \ldots, k \text { and } x_{i} \geqslant v(i) \text { for all } i \in N\right\} .
\end{aligned}
$$

The functions $F=\left\{F_{S}\right\}_{S \subset N, S \neq N, \varnothing}$ are the excess functions as before. The nucleolus which we find in this situation is denoted by $N(v, \mathscr{T}$ ) (cf. Owen 1977 and Wallmeier 1980). 
(c) Let $(N, v)$ be a cooperative game and $I^{*}(v)$ the set of pre-imputations, i.e., $\left\{x \in \mathbf{R}^{n} \mid \sum_{i \in N} x_{i}=v(N)\right\}$. Let $F=\left\{F_{S}\right\}_{S \subset N, S \neq N, \varnothing}$ be the excess functions as before. In this situation we cannot apply the theory of the preceding section immediately since $I^{*}(v)$ is not compact. But after one step in the construction of Theorem 1 we find a compact $\Pi_{1}$ for

$$
\Pi_{1} \subset\left\{x \in I^{*}(v) \mid v(i)-x_{i} \leqslant w_{1} \text { for all } i \in N\right\} .
$$

The nucleolus of this pair $\left(I^{*}(v), F\right)$ is called the pre-nucleolus $N^{*}(v)$ of the game (cf. Davis and Maschler 1965, Sobolev 1975).

(d) Let $(N, v)$ be a cooperative game and $w: 2^{N} \rightarrow \mathbf{R}_{++}$a weighting map. If we take $\Pi=I(v)$ and

$$
F=\left\{F_{S}: x \in I(v) \rightarrow((v(S)-x(S)) w(S))\right\}_{S \subset N, S \neq N, \varnothing}
$$

then we find the weighted nucleolus $N_{w}(v)$. In the literature the weights $w(S)=|S|^{-1}$ (cf. Grotte 1970) and $w(S)=f(|S|)^{-1}$ with $f:\{0,1,2, \ldots,|N|\} \rightarrow \mathbf{R}_{++}$increasing (cf. Wallmeier 1980) are mentioned.

Of course Examples 2(a) through (d) can be combined to, for example, $N_{w}^{*}(v, \mathscr{T})$, the weighted pre-nucleolus of a cooperative game $(N, v)$ with coalition structure $\mathscr{T}$.

REMARK. From Theorem 1 of $\S 1$ we infer immediately that all these nucleoli are nonempty and convex. Since, furthermore, the function $F$ is constant on the nucleolus $N(\Pi, F)$ we find in Examples 2(a)-(d) one-point sets (consider the functions $F_{\{i\}}$, $i \in N)$.

REMARK. In $\S 4$ we shall introduce excess games which reduce Examples 2(a)-(d) to special cases of Example 1.

2. The nucleolus $N(A)$ of a matrix game $A$. In this section we discuss the nucleolus of a matrix game. It turns out that the procedure described in the proof of Theorem 1 is just the Dresher procedure (cf. Dresher 1961). From this result and a theorem of van Damme (1983) we conclude that the nucleolus of a matrix game is the set of (weakly) proper equilibria of the game.

First we recall shortly the main well-known facts about matrix games. The value of a matrix game $A$ is given by

$$
\operatorname{val}(A):=\max _{p \in \Delta_{I}} \min _{j \in N} p A e_{j}=\min _{q \in \Delta_{I I}} \max _{i \in M} e_{i} A q .
$$

The set of optimal strategies of player I is defined by

$$
O_{I}(A):=\left\{p \in \Delta_{I} \mid p A e_{j} \geqslant \operatorname{val}(A) \text { for all } j \in N\right\} .
$$

Similarly the set of optimal strategies of player II is given by

$$
O_{I I}(A):=\left\{q \in \Delta_{I I} \mid e_{i} A q \leqslant \operatorname{val}(A) \text { for all } i \in M\right\} .
$$

Further we denote the carrier of a strategy $p \in \Delta_{I}$ by $C_{I}(p):=\left\{i \in M \mid p_{i}>0\right\}$, the carrier of the set of optimal strategies of player $I$ by $C_{I}(A):=\cup_{p \in O_{I}(A)} C_{I}(p)$ and the equalizer set

$$
\left\{i \in M \mid e_{i} A q=\operatorname{val}(A) \text { for all } q \in O_{I I}(A)\right\}
$$

by $E_{I}(A)$. The carriers $C_{I I}(q), C_{I I}(A)$ and the equalizer $E_{I I}(A)$ are defined similarly. 
A theorem of Bohnenblust, Karlin and Shapley (1950) (cf. also Gale and Sherman $1950)$ states the equality of $C_{I}(A)$ and $E_{I}(A)$ and of $C_{I I}(A)$ and $E_{I I}(A)$.

The procedure described in Dresher (1961) constructs from a matrix game $A$ a sequence of matrix games $A^{k}$ in the following way:

We start with the matrix game $A^{1}=A$. In the next game $A^{2}$ player I has the pure strategies $M \backslash C_{I}(A)$, player II has the strategy space $O_{I I}(A)$ and the payoffs are $e_{i} A q, e_{i} \notin C_{I}(A)$ and $q \in O_{I I}(A)$. If $A^{k-1}$ has been defined and $C_{I}\left(A^{k-1}\right) \neq \Delta_{I}^{k-1}$ then the pure strategies of player I in the game $A^{k}$ are the pure strategies of player I not used in the optimal strategies of the preceding games and the set of strategies of player II is the set $O_{I I}\left(A^{k-1}\right)$. After finitely many, say $k$, steps the set $M$ is exhausted and the set of Dresher-optimal strategies of player II is the set $O_{I I}\left(A^{k}\right)$. Let $b^{k}$ be the set of all pure strategies of player I used in the first $k$ steps of the Dresher procedure. Then

$$
\operatorname{val}\left(A^{k}\right)=\min _{q \in O_{I I}\left(A^{k-1}\right)} \max _{i \notin \mathscr{C}^{k-1}} e_{i} A q=\min _{q \in O_{I I}\left(A^{k-1}\right)} \max _{i \notin \mathscr{C}^{k-1}} F_{i}(q)
$$

If we compare this process with the construction of $\S 1$ we see immediately that $\Pi_{k}=O_{I I}\left(A^{k}\right), w_{k}=\operatorname{val}\left(A^{k}\right)$ and $\mathscr{B}_{k}=\mathscr{b}^{k}$. This means that $N_{I I}(A)$ equals the set $D_{I I}(A)$ of Dresher-optimal points of player II. Van Damme (1983) has proved that the set $D_{I}(A) \times D_{I I}(A)$ coincides with the set of (weakly) proper equilibria of the matrix game and therefore we find

THEOREM 2. The nucleolus of a matrix game is the set of proper equilibria of the game.

COROLlaRY. The set of proper equilibria of a matrix game is convex and exchangeable.

Remark. The set of perfect equilibria doesn't have this property (cf. Borm et al. 1988).

3. A balancedness criterion for the nucleolus of a matrix game. In this section we prove a balancedness result for the nucleolus of a matrix game.

Let $A$ be an $m \times n$-matrix game. The following lemma gives an alternative description of the data $\Pi_{k}, \mathscr{B}_{k}$ and $w_{k}$ occurring in the Dresher procedure of $\S 2$.

For $k=1, \ldots, s$ we define an $m \times n$-matrix $A^{(k)}$ with $i$ th row

$$
A_{i}^{(k)}= \begin{cases}A_{i}+\left(w_{1}-w_{k(i)}\right) e_{N} & \text { if } i \in \mathscr{B}_{k-1}, \\ A_{i}+\left(w_{1}-w_{k}\right) e_{N} & \text { if } i \notin \mathscr{B}_{k-1}\end{cases}
$$

This sequence of games $\left\{A^{(k)}\right\}$ has the advantage that the strategy sets of the players are the same for all games $A^{(k)}$.

By a simple induction argument one can prove

LEMMA 3. For $k=1, \ldots, s$ we have

(a) $\Pi_{k}=O_{I I}\left(A^{(k)}\right)$.

(b) $\operatorname{val}\left(A^{(k)}\right)=\operatorname{val}\left(A^{(1)}\right)=w_{1}$.

(c) $\mathscr{B}_{k}=E_{I}\left(A^{(k)}\right)=C_{I}\left(A^{(k)}\right)$.

To formulate the main result of this section we need the following definition.

We call a subset $R \subset M$ balanced with respect to $A$ on a set $C \subset N$ if there are a vector $p \in \mathbf{R}_{+}^{n}$, a vector $r \in \mathbf{R}_{+}^{n}$ and a real number $\alpha \in \mathbf{R}$ such that $p A-r=\alpha e_{N}$, $C_{I}(p)=R$ and $C_{I I}(r) \cap C=\varnothing$. 
For $q \in \Delta_{I I}$ we define for all $t \in \mathbf{R}$

$$
\mathscr{B}_{t}(q \mid A)=\left\{i \in M \mid F_{i}(q)=e_{i} A_{q} \geqslant t\right\}
$$

Then we have the following characterization of elements of $N_{I I}(A)$ (and of $N_{I}(A)$ ).

THEOREM 4. A strategy $q \in \Delta_{I I}$ is an element of the nucleolus $N_{I I}(A)$ if and only if for all $t \in \mathbf{R}$ the set $\mathscr{B}_{t}(q \mid A)$ is balanced with respect to $A$ on the set $C_{I I}(q)$. A similar result holds for the nucleolus $N_{I}(A)$.

Proof. $\Rightarrow$ Suppose that $q \in N_{I I}(A)$. Then $q \in \Pi_{k}=O_{I I}\left(A^{(k)}\right)$ for all $k=$ $1, \ldots, s$ (by Lemma 3). Hence, there is a strategy $p \in \Delta_{I}$ such that $C_{I}(p)=C_{I}\left(A^{(k)}\right)$ $=\mathscr{B}_{k}$ (by Lemma 3) and $r \in \mathbf{R}_{+}^{N}$ such that

$$
p A^{(k)}-r=\operatorname{val}\left(A^{(k)}\right) e_{N} \text { with } C_{I I}(r) \cap C_{I I}(q)=\varnothing .
$$

Then

$$
p A-r=\left\{\operatorname{val}\left(A^{(k)}\right)-\sum_{i \in \mathscr{B}_{k}} p_{i}\left(w_{1}-w_{k(i)}\right)\right\} e_{N} .
$$

This means that $\mathscr{B}_{k}$ is balanced with respect to $A$ on $C_{I I}(q)$.

We have to prove that for every $t \in \mathbf{R}$ there is an index $k$ such that $\mathscr{B}_{t}(q \mid A)=\mathscr{B}_{k}$. Let $t_{0}$ be the largest value of $t$ such that $\mathscr{B}_{t}(q \mid A) \neq \mathscr{B}_{0}, \mathscr{B}_{1}, \ldots, \mathscr{B}_{s}$ and suppose that $w_{k+1}<t_{0} \leqslant w_{k}$. There is an index $j_{0} \in \mathscr{B}_{t_{0}}(q \mid A)$ such that $F_{j_{0}}(q)=t_{0}$ (for, otherwise, $t_{0}$ is not maximal!). Since $F_{j_{0}}(q) \in\left\{w_{1}, \ldots, w_{s}\right\}$, we find $t_{0}=w_{k}$ and $\mathscr{B}_{t_{0}}(q \mid A) \supset \mathscr{B}_{k} \neq M$.

Suppose that $j \in \mathscr{B}_{t_{0}}(q \mid A)$ and $j \notin \mathscr{B}_{k}$. This means that $F_{j}(q) \geqslant t_{0}=w_{k}$ and, therefore, $g_{k+1}(q) \geqslant w_{k}$, i.e., $q \notin \Pi_{k+1}$ in contradiction with $q \in N_{I I}(A)$.

$\Leftarrow$ Suppose that $q \in \Delta_{I I}$ satisfies the balancedness conditions and suppose that $q \in \Pi_{k-1} \backslash \Pi_{k}$. Then there is an index $j_{0} \notin \mathscr{B}_{k-1}$ with $w_{k}<F_{j_{0}}(q):=t \leqslant w_{k-1}$.

From the balancedness of $\mathscr{B}_{t}(q \mid A)$ we infer that there is a strategy $p \in \Delta_{I}$ with $C_{I}(p)=\mathscr{B}_{t}(q \mid A)$, a vector $r \in \mathbf{R}_{+}^{N}$ with $C_{I I}(r) \cap C_{I I}(q)=\varnothing$ and a real number $\alpha \in \mathbf{R}$ such that $p A-r=\alpha e_{N}$. Then

$$
p A^{(k)}-r=\left\{\alpha+\sum_{i \in C_{I}(p)} p_{i}\left(w_{1}-w_{k(i)}\right)\right\} e_{N}=: \bar{\alpha} e_{N}
$$

- This means that $w_{1}=\operatorname{val}\left(A^{(k)}\right) \geqslant \bar{\alpha}$ and $p A^{(k)} q=\bar{\alpha}$.

- For each index $j \in \mathscr{B}_{t}(q \mid A)$ we have $e_{j} A q \geqslant t$ and $e_{j} A^{(k)} q \geqslant t+\left(w_{1}-w_{k}\right)>w_{1}$, if moreover, $j \notin \mathscr{B}_{k-1}$. For $i \in \mathscr{B}_{k-1}$ we have $e_{i} A^{(k)} q=w_{1}$ because $q \in \Pi_{k-1}$. So, $p A^{(k)} q>w_{1}$ since $C_{I}(p)=\mathscr{B}_{t}(q \mid A)$ and, in particular, $p_{j_{0}}>0$.

The contradiction between these two inequalities shows that the assumption $q \in \Pi_{k-1} \backslash \Pi_{k}$ is false. QED

4. The Kohlberg criterion for nucleoli of cooperative games. In this section we show that the nucleolus concepts for cooperative games can be found as the set of Dresher strategies (for player II) $N_{I I}(A)$ for well-chosen matrix games $A$. This enables us to 'translate' the theorem of the foregoing section into balancedness theorems for the nucleolus, prenucleolus and nucleolus of games with coalition structure. 
We start with a rather general situation. Let $\Pi \subset \mathbf{R}^{n}$ be a polytope (i.e., the convex hull of finitely many points) and let $F_{i}, i \in M$ be affine functions. Suppose that $E_{j}$, $j=1, \ldots, l$ are the extreme points of $\Pi$ and let $\Delta$ be the standard simplex in $\mathbf{R}^{l}$. Let $\phi: \Delta \rightarrow \Pi$ be the affine map $\phi(q)=\sum_{j=1}^{l} q_{j} E_{j}$. Then we can define the matrix (game) $A$ by $A_{i j}:=F_{i}\left(E_{j}\right)$ for all $i \in M$ and $j=1, \ldots, l$. Each point $x \in \Pi$ can be written as a convex expression in the extreme points $E_{j}, j=1, \ldots, l$ and we say that $E_{j}$ is in the carrier $(C(x))$ of a point $x \in \Pi$ if it is possible to use the extreme point in a convex expression of $x$. Note that for every point $x \in \Pi$ there is a point $q \in \Delta$ such that $\phi(q)=x$ and $C(x)=C(q)$.

It is an immediate consequence of the following lemma that $N_{I I}(A)=\phi^{-1} N(\Pi, F)$.

LeMmA 5. If $\Lambda: \Pi^{\prime} \rightarrow \Pi$ is an affine map onto $\Pi$ and $F$ is a finite set of continuous convex functions on $\Pi$, then $N\left(\Pi^{\prime}, F \circ \Lambda\right)=\phi^{-1} N(\Pi, F)$.

The proof of this lemma is straightforward and is therefore omitted.

From this result and Theorem 4 we find the following statements: $x \in N(\Pi, F) \Leftrightarrow$ $\phi^{-1}(x) \subset N_{I I}(A) \Leftrightarrow$ the set $\mathscr{B}_{t}(q \mid A)$ is balanced on $C(q)$ with respect to the matrix $A$ for all $q \in \phi^{-1}(x)$ and all real numbers $t$. Note that $\mathscr{B}_{t}(q \mid A)=\left\{i \in M \mid F_{i}(x)=\right.$ $\left.e_{i} A q \geqslant t\right\}=: \mathscr{B}_{t}(x \mid \Pi)$ if $q \in \phi^{-1}(x)$. Hence we find the following provisional result:

Let $x$ be a point of $\Pi$. Then $x \in N(\Pi, F)$ if and only if for all real numbers $t \in \mathbf{R}$ there are positive real numbers $\left\{p_{i}\right\}_{i \in \mathscr{B}_{t}(x \mid \Pi)}$ and a real number $\alpha$ such that

$$
\sum_{i \in \mathscr{B}_{t}(x \mid \Pi)} p_{i} F_{i}\left(E_{j}\right) \geqslant \alpha
$$

with equality if $E_{j} \in C(x)$.

Let $(N, v)$ be a cooperative game. We assume, for simplicity, that the game is zero-normalized i.e. $v(i)=0$ for all $i \in N$. Let $w: 2^{N} \rightarrow \mathbf{R}_{++}$be a weight function. Let $\Pi$ be a convex polytope in $\mathbf{R}^{N}$ with extreme points $\left\{E_{j}\right\}_{j=1, \ldots, l}$. Let $\left\{F_{S}\right\}_{S \neq \varnothing, N}$ be the set of (weighted) excess functions of the game $(N, v)$. In this case we call the matrix game $A$ defined in the same way as before the excess game of the game $(N, v)$ with respect to the set $\Pi$. In this situation equivalence (4.1) becomes:

If $x \in \Pi$, then the following statements are equivalent:

(1) $x \in N(\Pi, F)$ (in fact $x=N(\Pi, F)$ ).

(2) for all real numbers $t \in \mathbf{R}$ there are positive numbers $\left\{p_{S}\right\}_{S \in \mathscr{B}_{t}(x \mid \Pi)}$ and a real number $\alpha$ such that

$$
\left\langle\sum_{S \in \mathscr{B}_{t}} p_{S} w(S) e_{S}, E_{j}\right\rangle \leqslant \sum_{S \in \mathscr{B}_{t}} p_{S} w(S) v(S)-\alpha=: \bar{\alpha} \quad \text { for all } j=1, \ldots, l
$$

where $\langle\cdot, \cdot\rangle$ denotes the inner product in $\mathbf{R}^{N}, \mathscr{B}_{t}$ is a shorthand notation for $\mathscr{B}_{t}(x \mid \Pi)$ and $E_{j}, j=1, \ldots, l$ are the extreme points of $\Pi$ as before. Furthermore, there is equality if $E_{j} \in C(x)$. If we substitute $y_{S}:=p_{S} w(S)$ we find

$$
\left\langle\sum_{S} y_{S} e_{S}, E_{j}\right\rangle \leqslant \bar{\alpha}
$$

with equality for $E_{j} \in C(x)$.

In the remaining part of this section we investigate the meaning of (4.3) for special choices of $\Pi$.

(1) (weighted) nucleolus: If $\Pi=\mathscr{I}(v)$, the map $\phi$ is very simple $q \mapsto v(N) q$ and (4.3) gives $\left(\Sigma_{S} y_{S} e_{S}\right)_{j} \leqslant v(N)^{-1} \bar{\alpha}$ with equality if $x_{j}>0$. Since $\Sigma_{S} y_{S} e_{S}$ has at least 
one positive coordinate the number $v(N)^{-1} \bar{\alpha}>0$. After normalization we find the well-known results of Kohlberg (1971) and Wallmeier (1980).

(2) prenucleolus: Let $R$ be a real number such that $R>\max v(S)-|S| /|N| v(N)$ and $\Pi=\left\{x \in \mathscr{I}^{*}(v) \mid x_{i}+R \geqslant 0\right.$ for all $\left.i \in N\right\}$. Then the nucleolus $N(\Pi, F)$ is not on the boundary of $\Pi$ for a point on the boundary has at least one coordinate $x_{i}=-R$ and therefore maximal excess $\geqslant R$ but the point $z:=v(N) /|N| e_{N}$ has maximal excess $<R$. If we substitute $E_{j}=-R(1,1, \ldots, 1)+(v(N)+|N| R) e_{j}$ and $\overline{\bar{\alpha}}:=(v(N)+|N| R)^{-1}\left(\bar{\alpha}+R \Sigma_{S}|S| y_{S}\right)$ we find, since $C(x)=\left\{E_{j} \mid j \in N\right\}$,

$$
\sum_{S \in \mathscr{B}_{t}} y_{S} e_{S}=\overline{\bar{\alpha}} e_{N}
$$

After normalization $(\overline{\bar{\alpha}}>0)$ we find the balancedness result of Sobolev (1975).

(3) nucleolus for games with coalitional structure: For $\Pi=\mathscr{I}(v, \mathscr{T})$ the situation is a little more complicated since $\Pi$ is no longer a simplex but a product of simplices. The extreme points of $\Pi$ are the points $E_{J}:=v\left(T_{1}\right) e_{i(1)}+\cdots+v\left(T_{k}\right) e_{i(k)}$ where $J=\{i(1), \ldots, i(k)\}$ and $i(s)=J \cap T_{s}$ for $s=1, \ldots, k$. The carrier $C(x)$ of a point $x \in \Pi$ consists of these extreme points $E_{J}$ with $J=\{i(1), \ldots, i(k)\}$ and $x_{i(s)}>0$ for $s=1, \ldots, k$. Using equation (4.3) an easy calculation shows that there are real numbers $\alpha_{1}, \alpha_{2}, \ldots, \alpha_{k}$ such that

$$
\sum_{S \in \mathscr{D}_{i}} y_{S} e_{S} \leqslant \alpha_{1} e_{T_{1}}+\cdots+\alpha_{k} e_{T_{k}}
$$

with equality for the coordinates $j$ with $x_{j}>0$. This characterization of the nucleolus for games with coalitional structure can be found in Wallmeier (1980) (cf. also Owen 1979). by

If we call $\mathscr{B} \mathscr{T}$-balanced on $C(x)$ if (4.5) holds then we can summarize our results

Theorem 5. (a) (Kohlberg 1971) An imputation $x \in \mathscr{I}(v)$ is the (weighted) nucleolus of the game $(N, v)$ if and only if the collections $\mathscr{B}_{t}(x \mid v,(w))$ are balanced on $C(x)$ for all $t \in \mathbf{R}$ with $\mathscr{B}_{t}(x \mid v,(w)) \neq \varnothing$.

(b) (Sobolev 1975) A pre-imputation $x \in \mathscr{I}^{*}(v)$ is the prenucleolus of a game $(N, v)$ if and only if the collections $\mathscr{B}_{t}(x \mid v)$ are balanced for all $t \in \mathbf{R}$ where the collection is nonempty.

(c) (Owen 1977, Wallmeier 1980) An element $x \in \mathscr{I}(v, \mathscr{T})$ is the nucleolus of the game $(N, v)$ with coalition structure $\mathscr{T}$ if and only if the collections $\mathscr{B}_{t}(x \mid v)$ are $\mathscr{T}$-balanced for all relevant real numbers $t \in \mathbf{R}$.

REMARK. The nucleolus of a matrix game is a really more general concept than the nucleolus of a cooperative game. Some properties remain true (e.g., the balancedness results, cf. Theorem 4) but other properties become false (no longer a one-point concept, (semi-)continuity). Therefore there is little hope that one may succeed to prove our Theorem 4 from Kohlberg's result.

5. Some properties of the nucleoli of cooperative games. In this section we give another proof of some properties of the nucleoli of cooperative games.

(1) It is well known in the literature (cf. Maschler et al. 1979) that the prenucleolus and the nucleolus of a cooperative game are the same if the game is 0 -monotonic, i.e., if the 0-normalization of the game is monotonic. We shall give an alternative proof. 
Proof. Let $v$ be a 0-monotonic game and $x \in I^{*}(v) \backslash I(v)$. Then $x_{i}<v(i)$ for at least one index $i \in N$. Let $t \in \mathbf{R}$ be the largest number such that $\mathscr{B}_{t}(x \mid v) \neq \varnothing$. We prove that every element $S \in \mathscr{B}_{t}(x \mid v)$ contains player $i$. This follows from the fact that

$$
v(S \cup\{i\})-x(S \cup\{i\}) \geqslant v(S)-x(S)+v(i)-x_{i}>v(S)-x(S)
$$

for all $S \subset N \backslash i$ (the inequality is a consequence of the 0 -monotonicity of the game).

So, the collection $\mathscr{B}_{t}(x \mid v)$ cannot be balanced and $x \neq N^{*}(v)$. Therefore, $N^{*}(v)$ $\in I(v)$ and $N^{*}(v)=N(v)$. QED

(2) The prenucleolus of a game satisfies the reduced game property (cf. Sobolev 1975 and Davis and Maschler 1965). We shall reprove this property using the results of the preceding sections.

Let $(N, v)$ be a cooperative game, $x \in I^{*}(v)$ and $T \subset N$ a nonempty coalition. The reduced game $v_{x, T}$ is defined by

$$
\begin{aligned}
v_{x, T}(S):=\max _{R \cap T=\varnothing} v(S \cup R)-x(R) & \\
& \quad \text { if } S \subset T, S \neq T \text { and } v_{x, T}(T):=v(N)-x(N \backslash T) .
\end{aligned}
$$

We shall reprove the following property of the prenucleolus (reduced game property).

If $x$ is the prenucleolus of a game $(N, v)$ and $T$ is a nonempty coalition, then $N^{*}\left(v_{x, T}\right)=x_{\mid T}$ (the restriction of $x$ to $\left.T\right)$.

Proof. Note that the excess $e\left(S, x_{\mid T} \mid v_{x, T}\right)=\max _{R \cap T=\varnothing} e(S \cup R, x \mid v)$. This means that, for all $t \in \mathbf{R}, \mathscr{B}_{t}\left(x_{|T|} \mid v_{x, T}\right)=\mathscr{B}_{t}(x \mid v) \cap T$. From Theorem 5 we infer that $\mathscr{B}_{t}(x \mid v)$ is a balanced collection. Then $\mathscr{B}_{t}\left(x_{\mid T} \mid v_{x, T}\right)$ is balanced on $T$ for all $t \in \mathbf{R}$. Using Theorem 5 again we find that $x_{\mid T}$ is the prenucleolus of the reduced game $v_{x, T}$. QED

(3) In the literature there are several places where it is proved that the nucleolus or prenucleolus with or without weights and coalition structures are continuous functions of the data. On the other hand it is known that the set of proper equilibrium points of a matrix game is not upper semicontinuous. In this section we shall investigate where the difference comes from and reprove the continuity of the nucleoli.

We start with an example showing the discontinuity of the map $A \rightarrow N_{I I}(A)$. Take, for $n \in \mathbf{N}$,

$$
A_{n}:=\left(\begin{array}{rr}
0 & \frac{1}{n} \\
0 & -1
\end{array}\right) \rightarrow A:=\left(\begin{array}{rr}
0 & 0 \\
0 & -1
\end{array}\right)
$$

Note that in the matrices $A_{n}$ the first strategy of player I dominates the second strategy (weakly) and therefore the equilibrium point $\left(e_{1}, e_{1}\right)$ is the unique perfect (proper) equilibrium (cf. van Damme 1983). So, we have $N_{I I}\left(A_{n}\right)=\left\{e_{1}\right\}$. In the matrix $A$ the second strategy of player II dominates the first one and therefore $\left(e_{1}, e_{2}\right)$ is the unique proper equilibrium: $N_{I I}(A)=\left\{e_{2}\right\}$. Hence, there is no continuity in the point $A$.

Finally we reprove the continuity of the different type of nucleoli as function of the game and the weights.

Let $\left\{v_{n}\right\}_{n \in \mathrm{N}}$ be a sequence of cooperative games converging to the game $v$ and $\left\{w_{n}\right\}_{n \in \mathrm{N}}$ a sequence of weight functions converging to the weight function $w$. Further, 
let $x_{n}$ be the weighted nucleolus $N_{w_{n}}\left(v_{n}\right)$ for $n=1,2, \ldots$. Suppose that the sequence $\left\{x_{n}\right\}_{n \in \mathbf{N}}$ converges to $x \in \mathbf{R}^{N}$. We will prove that $x=N_{w}(v)$.

Let $\left\{A_{n}\right\}_{n \in \mathrm{N}}$ be the sequence of excess games of $\left\{v_{n}, w_{n}\right\}_{n \in \mathrm{N}}$ and $A$ the excess game of $v, w$. Then we have $A_{n} \rightarrow A$ and $q_{n} \rightarrow q$ if $q_{n}$ corresponds to $x_{n}$ and $q$ corresponds with $x$ under the affine map $\phi$. In order to prove that $q=N_{I I}(A)$ we try to show that $\mathscr{B}_{t}(q \mid A)$ is balanced on the carrier $C_{I I}(q)$ for all $t \in \mathbf{R}$.

Let $t \in \mathbf{R}$ and choose $\epsilon>0$ such that $\mathscr{B}_{t}(q \mid A)=\mathscr{B}_{t-2 \epsilon}(q \mid A)$.

Choose $N(\epsilon) \in \mathbf{N}$ such that, for all pure strategies $S$ of player I in the excess games and all $n \geqslant N(\epsilon),\left|e^{(S)} A_{n} q_{n}-e^{(S)} A q\right|<\epsilon$ and $C\left(q_{n}\right) \supset C(q)$. From this we infer

$$
S \in \mathscr{B}_{t}(q \mid A) \Rightarrow S \in \mathscr{B}_{t-\epsilon}\left(q_{n} \mid A_{n}\right) \Rightarrow S \in \mathscr{B}_{t-2 \epsilon}(q \mid A)=\mathscr{B}_{t}(q \mid A) .
$$

Then $\mathscr{B}_{t}(q \mid A)=\mathscr{B}_{t-\epsilon}\left(q_{n} \mid A_{n}\right)$ is balanced on $C\left(q_{n}\right)$ with respect to $A_{n}($ for $n \geqslant N(\epsilon))$. So, certainly $\mathscr{B}_{t}(q \mid A)$ is balanced on $C(q)$ with respect to $A_{n}$. In the case of cooperative games the words "with respect to $A_{n}$ " can be omitted and we find that $q=\phi(x)$ is in the nucleolus $N_{I I}(A)$ by Theorem 5. QED

For the prenucleolus and in games with coalitional structure the proof of the continuity goes along the same lines.

Note that the proof we gave above can be followed also for the nucleolus of matrix games up to the point

$$
\text { " } \mathscr{B}_{t}(q \mid A) \text { is balanced on } C(q) \text { with respect to } A_{n} \text { " }
$$

but now the addition "with respect to $A_{n}$ " is very important. Let us reconsider our counterexample. There we have $\mathscr{B}_{t}\left(e_{1} \mid A\right)=\left\{e_{1}, e_{2}\right\}$ for $t \leqslant 0$ and we can balance the rows of $A_{n}$ on $C\left(e_{1}\right)=\left\{e_{1}\right\}$ by

$$
\frac{n}{n+1}\left(0, \frac{1}{n}\right)+\frac{1}{n+1}(0,-1)=(0,0)
$$

but we cannot do the same for the limit matrix $A$ because

$$
p_{1}(0,0)+p_{2}(0,-1)=\left(0,-p_{2}\right)
$$

and the second coordinate is smaller than the first one if $p \in \Delta_{2}^{0}$.

Acknowledgements. We thank Peter Borm for his careful reading of this manuscript. By his cooperation the readability of this manuscript was greatly improved.

\section{References}

Aumann, R. J. and Drèze, J. H. (1974). Cooperative Games with Coalition Structure. Internat. J. Game Theory 3 217-237.

Bohnenblust, H. F., Karlin, S. and Shapley, L. S. (1950). Solutions of Discrete Two-Person Games. Ann. of Math. Studies 24 51-72.

Borm, P. E. M., Jansen, M. J. M., Potters, J. A. M. and Tijs, S. H. (1988). On the Structure of the Set of Undominated Equilibria in Bimatrix Games. Internal Report of NICI, University of Nijmegen, 88 NICI 09.

van Damme, E. E. C. (1983). Refinements of the Nash Equilibrium Concept. Springer Verlag, Berlin.

Davis, M. and Maschler, M. (1965). The Kernel of a Cooperative Game. Naval Res. Logist. Quart. 12 223-259.

Dresher, M. (1961). Games of Strategy. Prentice-Hall Inc., Englewood Cliffs, NJ, 71-73.

Gale, D. and Sherman, S. (1950). Solutions of Finite Two-Person Games. Ann. of Math. Studies 24 37-49. 
Grotte, J. H. (1970). Computation of and Observations on the Nucleolus, the Normalized Nucleolus and the Central Games. M.S. Thesis, Cornell University, Ithaca, NY.

Kohlberg, E. (1971). On the Nucleolus of a Characteristic Function Game. SIAM J. Appl. Math. 20 62-65. Maschler, M., Peleg, B. and Shapley, L. S. (1979). The Kernel and the Bargaining Set for Convex Games. Internat. J. Game Theory 1 73-93.

, Potters, J. A. M. and Tijs, S. H. (1991). The Abstract Nucleolus and the Reduced Game Property. Discussion paper, Department of Mathematics, University of Nijmegen.

Myerson, R. B. (1978). Refinements of the Nash Equilibrium Concept. Internat. J. Game Theory 7 73-80.

Owen, G. (1977). A Generalization of the Kohlberg Criterion. Internat. J. Game Theory 6 249-255.

Schmeidler, D. (1969). The Nucleolus of a Characteristic Function Game. SIAM J. Appl. Math. 17 $1163-1170$.

Sobolev, A. I. (1975). A Characterization of Optimality Principles in Cooperative Games by Functional Equations. (Russian) Math. Methods Social Sci. 6 94-151.

Wallmeier, E. (1980). Der f-Nukleolus als Lösungskonzept für n-Personenspiele in Funktionsform. Diplomarbeit, Universität Münster.

DEPARTMENT OF MATHEMATICS, UNIVERSITY OF NIJMEGEN, TOERNOOIVELD, 6525 ED NIJMEGEN, THE NETHERLANDS 